\title{
Clinical Pearl: \\ COVID-19 in the Neonatal Population: \\ Review of Current Literature
}

Kenneth Kellner, MD

\section{What is COVID-19?}

Many members of the Coronaviridae family exist, and usually, Coronaviruses cause mild respiratory disease or diarrhea. (1) Towards the end of 2019, COVID-19 emerged in local hospitals in Wuhan, China. Initial investigation suggested cases were associated with exposure at a local seafood market where many varieties of live animals were available for sale. On February 11, 2020, the World Health Organization (WHO) announced the standard format: Coronavirus Disease-2019 (COVID-19). The same day, the International Committee on Taxonomy of Viruses (ICTV) named the virus SARS-CoV-2. SARS-CoV-2 was first isolated in bronchoalveolar lavage fluid of three patients at a Wuhan hospital on December 30, 2019. (2)

SARS-CoV- 2 is a member of the $\beta$-CoV family. The Coronavirus (CoV) family are enveloped, positive-sense, single-stranded RNA viruses. Four subfamilies exist: $\alpha, \beta, \gamma$, and $\delta$. Human CoV infections are caused by $a$ and $\beta-C o V s$. SARS-CoV-2 is a member of the $\beta$-CoV family. (2)

The spike (S) protein of coronaviruses facilitates viral entry into target cells. Entry depends on the binding of the surface unit of the $S$ protein to a cellular receptor. SARS-CoV-2 utilizes angiotensinconverting enzyme 2 (ACE2) as the entry receptor. (1)

\section{Is COVID-19 vertically transmitted from mother to neonate?}

A systematic review published in May 2020 aimed at studying vertical transmission of SARS-CoV-2. A review of 22 studies comprising 83 neonates born to mothers diagnosed with COVID-19, both via cesarean delivery (CS) and vaginal delivery (VD), revealed three positive confirmed cases of SARS-CoV-2 in neonates via nasopharyngeal swab real-time polymerase chain reaction (RT-PCR). Another six patients had elevated virusspecific antibody levels but negative PCR testing. Without positive RT-PCR tests of amniotic fluid, placenta, or cord blood, there is a lack of evidence for intrauterine vertical transmission. In addition, there is no convincing evidence that CS is safer than VD. (3)

Another systemic review of 49 studies and 666 neonates demonstrated 28 infants $(28 / 666,4 \%)$ had confirmed COVID-19 infection. A total of 291 women delivered vaginally, and 8/292 $(2.7 \%)$ of these neonates tested positive for SARS-CoV-2. Three hundred and sixty-four women delivered via CS, and 20/374 $(5.3 \%)$ of these neonates tested positive. This study demonstrates neonatal COVID-19 infection is uncommon, usually asymptomatic, and the rate of infection is not significantly lower when babies are born via CS versus VD. Importantly, rate of neonatal infection does not appear to be significantly different comparing infants who were allowed to breastfeed, room-in with or come into contact the mother versus infants who were separated from mom or formula feed. (4)
"The National Registry for Surveillance and Epidemiology of Perinatal COVID-19 Infection (NPC-19) includes 176 centers in the United States thus far with an aim to monitor data and outcomes of mother/ infant dyads when mothers test positive for COVID-19."

The National Registry for Surveillance and Epidemiology of Perinatal COVID-19 Infection (NPC-19) includes 176 centers in the United States thus far with an aim to monitor data and outcomes of mother/infant dyads when mothers test positive for COVID-19. These data were last updated on June 5, 2020, and now includes 747 mother/infant dyads. Of 747 mothers who tested positive for COVID-19, 508 (68.6\%) were asymptomatic, $191(25.8 \%)$ were symptomatic, and $42(5.7 \%)$ were admitted for COVID-19 treatment. Twenty-five infants thus far have had at least one positive test for COVID-19. Of these, 14 were separated from mom after birth, and 11 were non-separated. Of 232 mothers with clinical illness, 182 infants were separated, and six were presumptive positive (infection rate of $3.3 \%$ ). Fifty infants both to mothers with clinical illness roomed in with mother, and 1 of these infants tested positive ( $2 \%$ infection rate). A total of 500 asymptomatic women tested positive for COVID-19, and 212 of these infants were separated from mom. Of these 212 separated infants, three tested positive (1.4\%). 288 infants born to asymptomatic women roomed in, and ten of the infants tested positive (3.4\%). (5)

As further data are published, the information gathered will likely lend more answers to the question of vertical transmission. Based on the data currently published, it is apparent that CS is not necessarily safer than VD. Likewise, the argument can be made that neonates rooming in with mother-whether mom is symptomatic or asymptomatic-do not infer a higher risk of infection versus infants who are separated.

\section{A Convincing Case Report of Potential Vertical Transmission:}

A recent case report suggests the possibility of vertical transmission. The infant was born to a 40-year-old woman with active SARS-CoV-2 infection in Toronto, Canada. Mother had a history of familial neutropenia, gestational diabetes and had frequent bacterial infections during pregnancy treated with antibiotics. She presented with myalgia, dry cough, and fever and tested positive for SARS-CoV-2 via reverse transcriptasepolymerase chain reaction (RT-PCR). This mother did not require respiratory support. Due to worsening maternal coagulopathy, urgent CS was performed at 35+5 weeks gestation. There was 
an artificial rupture of membranes at delivery, clear fluid, and delivery of a neonate with Apgar scores of 9 and 9 at one and five minutes, respectively, with a birth weight of $2.93 \mathrm{~kg}$. Contact and droplet precautions were observed in the operating room. Nasopharyngeal swabs of the neonate were positive on the day of birth, post-natal day of life (DOL) 2 , and DOL 7 . Neonate's plasma tested positive on DOL 4, and stool tested positive on DOL 7. Five random placenta specimens showed areas of infiltration by inflammatory cells and early infarction. (6)

In the same case, mother and newborn were transferred to a negative pressure room, mom attempted breastfeeding with precautions, and the neonate was kept two meters away from mom between feeds. The infant was then transferred to the Neonatal Intensive Care Unit (NICU) for hypoglycemia and neutropenia. The infant was eventually discharged home on DOL 4. In this case, congenital infection is supported by the fact that the neonate was not in contact with the mother's vaginal secretions, membranes were intact before birth, and there was no skin-toskin contact with mother prior to collection of the first PCR test. Of note, mother tested positive via nasopharyngeal swab and also tested positive in breast milk and vaginal secretions. 6 If this case truly represents vertical transmission, it is likely the mother's immunocompromised state and disseminated infection played a significant role in the transmission of SARS-CoV-2 to the neonate.

\section{Can COVID-19 be Transmitted Via Breast Milk?}

A study from Germany in May 2020 examined breast milk from two nursing mothers infected with SARS-CoV-2. After nipple disinfection, milk was collected with pumps and tested for viral loads via RT-PCR. Four samples from mother number one tested negative. SARS-CoV-2 RNA was detected in the milk of mother number two at days 10,12 , and 13 after delivery. Subsequent samples collected from mother two were negative. Both newborn one and two tested positive for COVID-19 on DOL 8 and DOL 11 , respectively. It is unclear whether the newborn born to mother number two was infected via mother's breast milk or via another mode of transmission. (7) Further studies are needed in which mother's milk is tested for SARS-CoV-2, and the neonate is tested and followed clinically over time in order to determine if breast milk is a source of vertical transmission from mother to neonate.

\section{Is the Placenta Affected by Maternal COVID-19 Infection?}

A recent study from Northwestern University examined placentas of 16 mothers infected with SARS-CoV-2. Fifteen were live births in the third trimester, and one was delivered in the second trimester after intrauterine fetal demise. Compared to controls, third trimester placentas were significantly more likely to show maternal vascular malperfusion (MVM). MVM is a pattern of placental injury indicating abnormalities in oxygenation in the intervillous space and is associated with adverse perinatal outcome. (8)

\section{Does COVID-19 Adversely Affect Neonates?}

A recent case study reports a term neonate born after an uncomplicated pregnancy. On the second day after delivery, the mom developed a fever and tested positive for SARS-CoV-2 via PCR. On the same day, the infant also tested positive. Initially, the infant was asymptomatic, but after 48 hours of isolation in the same room as mother, the baby developed perioral cyanosis and a poor suck without other signs of respiratory distress. Complete blood count and C-reactive protein were reassuring. The baby's oxygen saturation was $88 \%$ on room air; the remainder of vital signs were within normal limits. Arterial blood gases demonstrated hypoxemia. An echocardiogram excluded a cardiac cause of cyanosis. The infant was started on empiric ampicillin and gentamicin, blood culture resulted showed no growth, and the infant tested negative for other respiratory viruses via PCR. The chest radiograph revealed mild ground-glass opacities. The infant required high flow nasal cannula $\left(\mathrm{FiO}_{2} 30 \%\right)$. Weaning to room air was accomplished after 50 hours of NICU admission. This case indicates that infection with SARS-CoV-2 may result in significant hypoxemia in the neonate. (9)

This update of clinical information regarding aspects of the transmission and effects of COVID-19 infection in mothers and their newborn infants reflects the almost daily availability of new information for providers.

\section{References}

1. Hoffmann M, Kleine-Weber H, Schroeder S, et al. SARSCoV-2 Cell Entry Depends on ACE2 and TMPRSS2 and Is Blocked by a Clinically Proven Protease Inhibitor. Cell. 2020;181(2):271-280.e8. doi:10.1016/j.cell.2020.02.052

2. Jin $Y$, Yang $H$, Ji $W$, et al. Virology, Epidemiology, Pathogenesis, and Control of COVID-19. Viruses. 2020;12(4):372. Published 2020, March 27. doi:10.3390/ v12040372

3. Yang Z, Liu Y. Vertical Transmission of Severe Acute Respiratory Syndrome Coronavirus 2: A Systematic Review [published online ahead of print, 2020 May 13]. Am J Perinatol. 2020;10.1055/s-0040-1712161. doi:10.1055/s-0040-1712161

4. Walker KF, O'Donoghue K, Grace $N$, et al. Maternal transmission of SARS-COV-2 to the neonate, and possible routes for such transmission: A systematic review and critical analysis [published online ahead of print, 2020 June 12]. BJOG. 2020;10.1111/1471-0528.16362. doi:10.1111/14710528.16362

5. National Registry for Surveillance and Epidemiology of Perinatal COVID-19 Infection. American Academy of Pediatrics

6. Kirtsman M, Diambomba Y, Poutanen SM, et al. Probable congenital SARS-CoV-2 infection in a neonate born to a woman with active SARS-CoV-2 infection [published online ahead of print, 2020 May 14]. CMAJ. 2020;cmaj.200821. doi:10.1503/cmaj.200821

7. Groß R, Conzelmann C, Müller JA, et al. Detection of SARSCoV-2 in human breastmilk. Lancet. 2020;395(10239):17571758. doi:10.1016/S0140-6736(20)31181-8

8. Shanes ED, Mithal LB, Otero S, Azad HA, Miller ES, Goldstein JA. Placental Pathology in COVID-19. Am J Clin Pathol. 2020;154(1):23-32. doi:10.1093/ajcp/aqaa089

9. Sinelli M, Paterlini G, Citterio M, Di Marco A, Fedeli T, Ventura ML. Early Neonatal SARS-CoV-2 Infection Manifesting With Hypoxemia Requiring Respiratory Support. Pediatrics. 2020;146(1):e20201121. doi:10.1542/peds.2020-1121

The author has no conflicts to disclose 


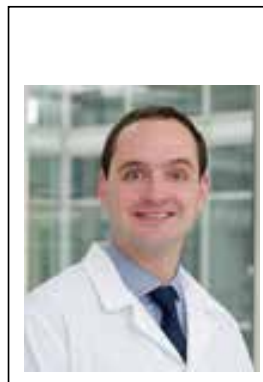

Corresponding Author

Kenneth Kellner, MD PGY5

Neonatal-Perinatal Medicine Fellow

University of Chicago

Comer Children's Hospital

Chicago, IL

Kellner, Kenneth [UCM] < Kenneth.Kellner@uchospitals.edu $>$

\section{Clinical Pearls are published monthly.}

Submission guidelines for "Clinical Pearls":

1250 word limit not including references or title page.

May begin with a brief case summary or example.

Summarize the pearl for emphasis.

No more than 7 references.

Please send your submissions to:

jhageman@peds.bsd.uchicago.edu

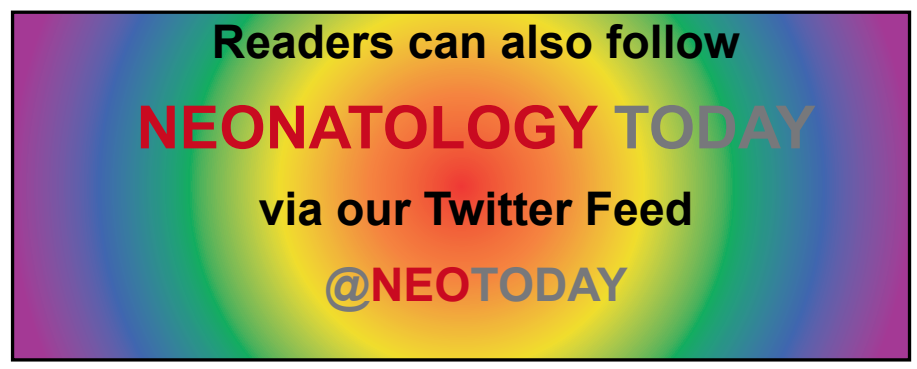

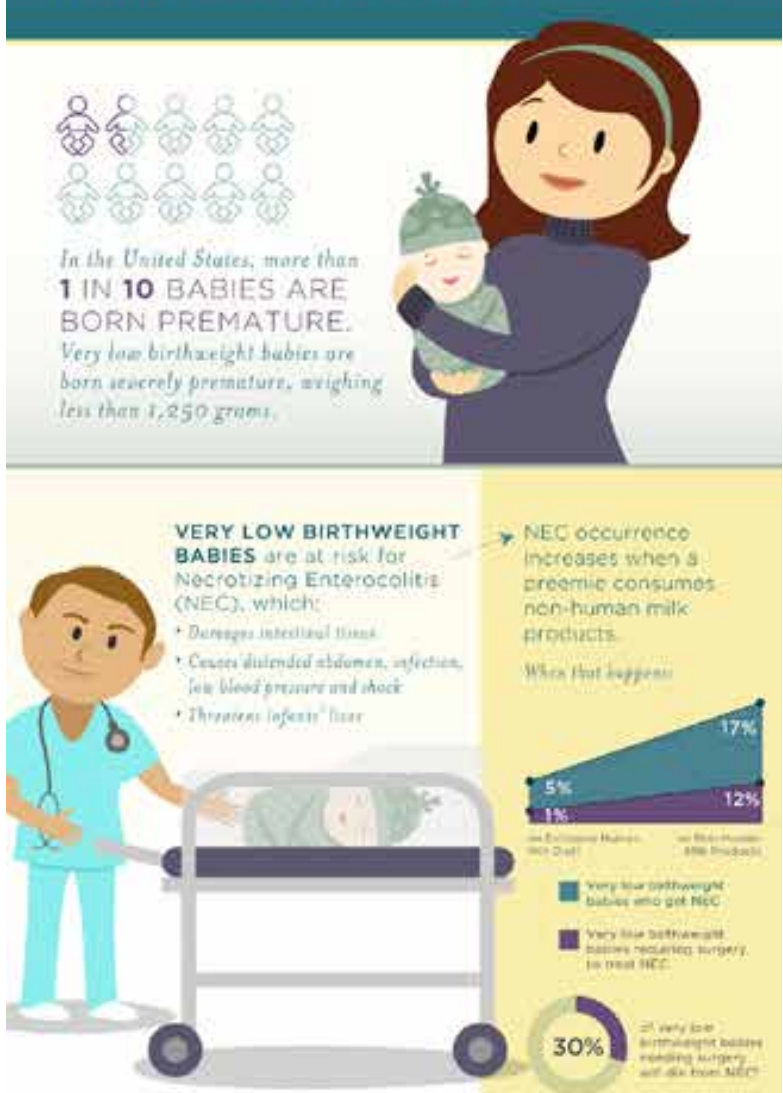

HOW TO HELP PREVENT NEC: EXCLUSIVE HUMAN MILK DIET

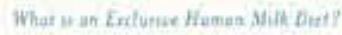
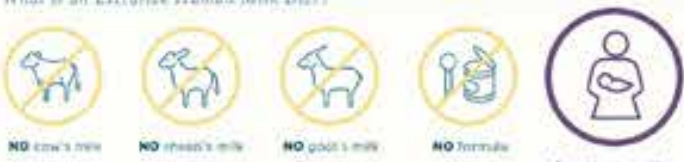
$\checkmark$ mothersma $\checkmark$ humian donor ner. $\checkmark$ nimasamelibaseli

Why IS An Exclusive Human Milk Dlet incottan?

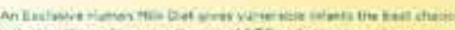

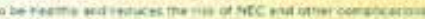

Wue n a fow dirthiceight bohy cas wees in EXCLUSIVE HUMAN MILK DIET

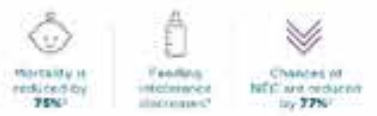

HUMAN MILK = MEDICINE

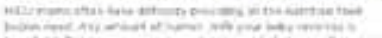

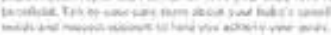

LEARN MORE ,

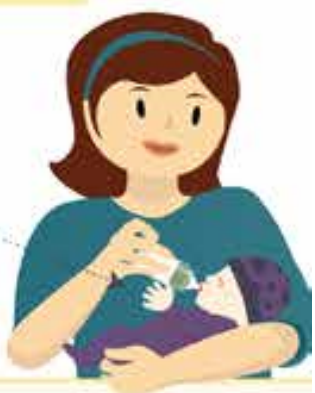

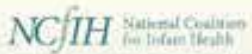

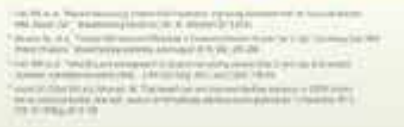

